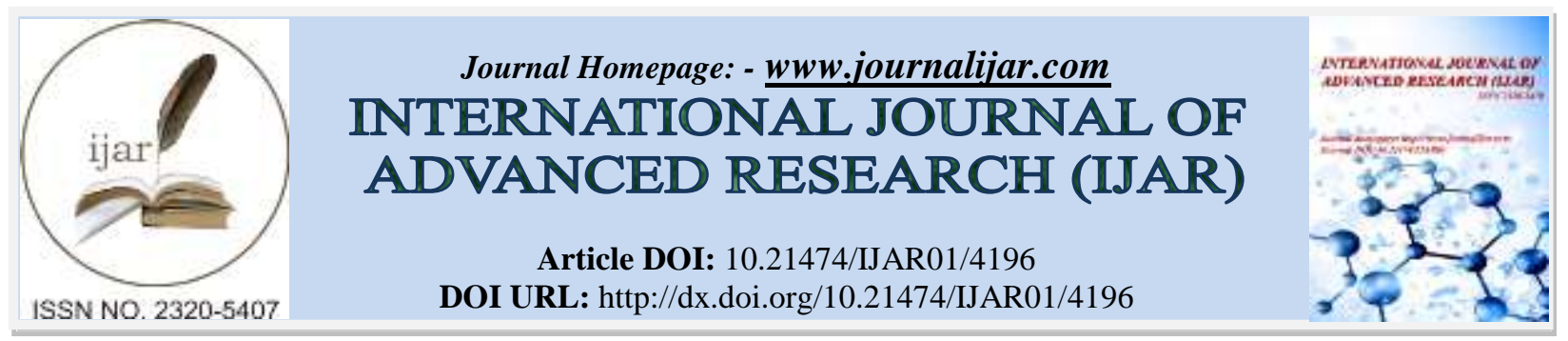

RESEARCH ARTICLE

\title{
CHEMICAL COMPOSITION OF ESSENTIAL OIL OF TEUCRIUM PSEUDOSCORODONIA SUBSP. BAETICUM, ENDEMIC PLANTOF NORTH OF MOROCCO.
}

\section{Nabila Tahoum ${ }^{1}$, Abdelhay Arakrak ${ }^{1}$, Mohamed Bakkali ${ }^{1}$, Sorel S. Jatunov ${ }^{2}$, Antonio G. Franconetti, ${ }^{2}$} Manuel G. Guillén ${ }^{2}$, Francisca C. Escribano ${ }^{2}$ and ${ }^{*}$ Amin Laglaoui ${ }^{1}$.

1. Department of Life Sciences, Faculty of Sciences and Technics of Tangier, Ziaten. BP: 416. Tangier, Morocco.

2. Department of Organic Chemistry, Faculty of Chemistry, University of Seville, c/ professor García González, s/n c.p: 41012. Seville, Spain.

\section{Manuscript Info}

Manuscript History

Received: 15 March 2017

Final Accepted: 17 April 2017

Published: May 2017

Key words:-

Teucrium pseudoscorodonia subsp. baeticumof North of Morocco; essential

oil; monoterpenes; alkaloids; morpholine.

\section{Abstract}

The chemical composition of essential oil of dry leaves of Teucrium pseudoscorodonia subsp.baeticum of North of Morocco from Perdicaris forest was investigated by GC-MS analysis. The detected components were aromatic compounds $(12.38 \%)$, oxygenated monoterpenes $(7.78 \%)$, fatty acids related compounds $(6.91 \%)$, alkaloids (2.12\%), flavonoids (1.82\%), and lactones $(0.62 \%)$ and the main components were N-Formylmorpholine (25.07 \%), morpholine, 4-acetyl- (17.61 \%) and 2-Oxabicyclo[2,2,2]octan-6-one, 1,3,3trimethyl- $(6.47 \%)$.

Copy Right, IJAR, 2017,. All rights reserved.

\section{Introduction:-}

North of Africa houses nearly 1700 species and subspecies endemic, more than $50 \%$ exist only in Morocco. The largest number is in Morocco and Algeria. The herbarium MPU (University of Montpellier 2) have a huge part, followed by the Museum P (National Museum of Natural History, Paris) and RAB (Scientific Institute, Rabat) with respectively 3054, 1059 and 933 exsiccata. Teucrium pseudoscorodonia subsp. Baeticum is an endemic plant of north of Morocco (El Oualidi J. and Khamar H., 2012) and Teucrium is the largest genera of the Lamiaceae in Morocco (Navarro T. and El Oualidi J. 1997. Synopsis of the Genus Teucrium L. (Lamiaceae) In Morocco.Acta Botanica Malacitana 22:187-203). Since the end of XVIII century, different authors have studied this genus; Desfontaines (1798), Ball (1878), Cosson (1873; 1881), Battandier (1917), Battanclier and Trabut (1890), Pau (1924), Litardierc and Maire (1921), Humbert (1924),Font Quer (1928; 1932), Emberger and Maire(1927; 1941), Maire (1929a, b; 1931; 1932;1933; 1939), Jahandiez and Maire (1934), Sennen (1936), Sauvage and Vindt (1955; 1965; 1967), Cohen (1956), El Oualidi (1987; 1991), El Oualidi and Ibn Tattou (1993), El Oualidi and Navarro (1995), Navarro and El Oualidi (1996) and El Oualidi and al. ( I 997a; 1997b).

$L=T$. pseudoscorodonio var. baeticum (Boiss. and Reuter) Maire in Jahand. and Maire, Cat. Pl. Maroc 3: 613 (1932); The genus Teucrium L. (Lamiaceae) comprises more than 300 species (J.A. Franco, Nova Flora de Portugal (Continente e Açores), II, Lisboa, 1984. B. Valdés, S. Talavera, E. Fernández-Galiano, Flora vascular de Andalucıa Ocidental, Ketres Editora, Barcelona, 1987). It is present in the Mediterranean basin ((Boiss. and Reuter)). (Tutin, T. G., Heywood, V. H., Burges, N. A., Moore, D. M., Valentie, D. H., and Webb, D. A. (1976). Flora Europaea (Vol. 3). Cambridge: Cambridge University Press. 
The specie of Teucrium pseudoscorodonia subsp. baeticum prefers broad-leaved forests, generally on acid soil, from sea level up to $1500 \mathrm{~m}$ (Pignatti, 1982).

Many species of Teucrium are known for their utilizationin traditional folk medicine and are claimed to exhibit interesting biological properties, e.g., hypoglycaemic, hypolipidemic, antipyretic, antiulcer and antibacterial (Nagao,Ito, Kohno, Kuroda, and Fujita, 1982; Autore et al., 1984; Gharaibeh, Elayan, andSalhab, 1988; Tariq, Ageel, Al-Yahya, Mossa, and Al-Said, 1989; Roman-Ramos, Flores-Saenz, Partida-Hernandez, Lara-Lemus, and AlarconAguilar,1991; Fernandez, Iglesias, and Villar del Fresno, 1997;Galati et al., 2000; Rasekh, KhoshnoodMansourkhani and Kamalinejad, 2001; Couladis, Tzakou, Verykokidou, and Harvala, 2003).

Teucrium species have been used as medicinal plants for more than 2000 years and some of them are still used in folk medicine as antispasmodic, tonic, antipyretic and antiseptic (M.M. Hassan, F.J. Muhtadi, A.A. Al-Badr, J. Pharm. Sci. 68 (1979), 800). This genus is chemically characterized by volatile constituents (A. VelascoNegueruela, M.J. Pérez-Alonso, Phytochemistry 29 (1990) 1165. C. Cavaleiro, L.R. Salgueiro, T. Antunes, I. Sevinate-Pinto, J.G. Barroso, Flav. Frag. J. 17 (2002) 287).

\section{Materials and methods:-}

The Teucrium pseudoscorodonia subsp. Baeticum was collected from The Perdicaris forest (Site of Biological and Ecological interest) in Tangier in Morocco. The site is located on the outskirts of the town of Tangier (Tangiers) at the western extremity of the southern side of the Straits of Gibraltar. Forest-cover is highest on the northern slopes between 80 and $240 \mathrm{~m}$ in altitude. The climate is hot and humid thermomediterranean.

The leaves of Teucrium pseudoscorodonia subsp. Baeticum were dried at room temperature then submitted to hydrodistillation to extract essential oil using Clevenger apparatus. The yield was $0.2 \%$. The mass spectrometry chromatography (GC-MS) (Department of Organic Chemistry, Seville, Spain) was carried out using Agilent Model 7890A coupled to Agilent 5975C inert MSD with Triple-Axis Detector and Agilent Technologies 7693 Auto sampler using split ratio 5:1 Agilent 5183-4647 liner $4 \mathrm{~mm}$ ID LPD and HP5 column (30 $\mathrm{m} \times 0.320 \mathrm{~mm} \times 0.25 \mu \mathrm{m})$. The column temperature was kept to $280{ }^{\circ} \mathrm{C}$ for $30 \mathrm{~min}$ then programmed to $300{ }^{\circ} \mathrm{C}$ at a rate $3{ }^{\circ} \mathrm{C} / \mathrm{min}$. Flow rate of helium as a carrier gas was $20 \mathrm{ml} / \mathrm{min}$.

Table 1:- GC-MS Composition of essential oil of Teucrium pseudoscorodonia subsp. baeticum of Morocco

\begin{tabular}{|c|c|c|c|}
\hline No. & Compounds & $\operatorname{Tr}(\min )$ & $\%$ \\
\hline 1 & 2-Butenal, 3-methyl- & 10.461 & 0.93 \\
\hline 2 & N-Formylmorpholine & 11.411 & 25.07 \\
\hline 3 & 2-Pentanone, 5-(acetyloxy)- & 11.846 & 0.16 \\
\hline 4 & 3-Heptanone, 2,6-dimethyl- & 13.053 & 0.29 \\
\hline 5 & Succinicacid, cyclohexylmethylisohexyl ester & 13.62 & 0.3 \\
\hline 6 & Morpholine, 4-acetyl- & 14.037 & 17.61 \\
\hline 7 & 2,2-Dimethylpentyl cyclohexanecarboxylate & 14.97 & 0.15 \\
\hline 8 & 2-n-Hexylcyclopentanone & 15.582 & 1.05 \\
\hline 9 & Cyclobutanecarboxylicacid, 2-tetrahydrofurylmethyl ester & 15.651 & 0.91 \\
\hline 10 & 3-Piperidinol, 6-methyl- & 16.263 & 2.44 \\
\hline 11 & 1-Heptadecyne & 17.007 & 0.3 \\
\hline 12 & Morpholine, 4-propionyl- & 17.207 & 0.5 \\
\hline 13 & Allyl Isothiocyanate & 19.399 & 0.5 \\
\hline 14 & Proline, 3-hydroxy-4-methyl- & 19.891 & 0.19 \\
\hline 15 & 1,1-Dodecanediol, diacetate & 20.011 & 0.25 \\
\hline 16 & trans-1,2-Dimethylsilacyclohexane & 20.166 & 0.24 \\
\hline 17 & Cyclohexanecarboxylicacid, 2-tetrahydrofurylmethyl ester & 20.492 & 2.2 \\
\hline 18 & 3-Piperidinone, 1-ethyl- & 20.766 & 2.12 \\
\hline 19 & Heptylcyclohexane & 23.479 & 6.02 \\
\hline 20 & Cyclohexanone, 2,6-dimethyl-2-(2-oxopropyl)- & 23.622 & 0.4 \\
\hline 21 & 2-Acetyl-6-methyl-2,3-dihydropyran & 23.816 & 1.82 \\
\hline 22 & 1,3-Hexadiene, 3-ethyl-2-methyl-,(Z)- & 23.999 & 0.77 \\
\hline 23 & 1,3-Hexadiene, 3-ethyl-2-methyl-,(Z)- & 24.280 & 0.38 \\
\hline 24 & Pentanoicacid, morpholide & 24.554 & 0.51 \\
\hline
\end{tabular}




\begin{tabular}{|c|c|c|c|}
\hline 25 & 2,6-Pyridinediamine & 24.715 & 2.9 \\
\hline 26 & 3-Methyl-2-butenoic acid, 2-ethylcyclohexyl ester & 25.086 & 3.75 \\
\hline 27 & 2-Oxabicyclo[2,2,2]octan-6-one, 1,3,3-trimethyl- & 25.470 & 6.47 \\
\hline 28 & 4-Hepten-3-one, 2,6-dimethyl- & 25.847 & 1.55 \\
\hline 29 & 2-Cyclopenten-1-one, 3-methoxy-4-methyl- & 26.265 & 0.86 \\
\hline 30 & Thiophene, 2-(2-methylpropyl)-4-Octen-3-one & 26.460 & 1.78 \\
\hline 31 & Phosphonofluoridic acid, ethyl-, 2-methylpentyl ester & 26.631 & 0.23 \\
\hline 32 & Cyclohexanone, 2-(1-methylethyl)- & 27.467 & 0.27 \\
\hline 33 & Silane, dimethyldi-2-propenyl- & 27.999 & 1.62 \\
\hline 34 & Hexanoicacid, morpholide & 28.56 & 0.4 \\
\hline 35 & 2-Chlorophenylhydrazine & 28.806 & 0.3 \\
\hline 36 & 2H-Pyran-2-one, 6-hexyltetrahydro- & 28.954 & 0.42 \\
\hline 37 & 1,3-Dioxolane, 4-[[(2-methoxy-4-octadecenyl)oxy]methyl]-2,2-dimethyl & 29.498 & 0.43 \\
\hline 38 & 2-Butyl-2,7-octadien-1-ol & 29.756 & 0.4 \\
\hline 39 & L-Alanine, N-L-alanyl- & 29.876 & 2.62 \\
\hline 40 & 2,6-Dimethyl-4-thiopyrone & 30.082 & 0.81 \\
\hline 41 & 1,3-Dioxolane, 4-[[(2-methoxy-4-octadecenyl)oxy]methyl]-2,2-dimethyl & 30.677 & 0.23 \\
\hline 42 & Piperidine, 3,5-dimethyl- & 32.033 & 0.52 \\
\hline 43 & Acetoxyaceticacid, morpholide & 32.388 & 0.19 \\
\hline 44 & 4,8,12,16-Tetramethylheptadecan-4-olide & 32.754 & 0.31 \\
\hline 45 & 5-t-Butyl-hexa-3,5-dien-2-one & 33.532 & 0.17 \\
\hline 46 & 4-Bromo-4-methyl-5-oxo-tetrahydro-furan-2-carboxylic acid & 33.807 & 0.17 \\
\hline 47 & trans-4a-Methyl-decahydronaphthalene & 34.940 & 0.37 \\
\hline 48 & Bicyclo[2,2,1]heptan-2-one, 1,7,7-trimethyl-, (,+/-,)- & 35.186 & 0.91 \\
\hline 49 & 4,8,12,16-Tetramethylheptadecan-4-olide & 36.605 & 0.21 \\
\hline 50 & Acetohydrazide, 2-(4-morpholyl)-N2-[(4-methylcyclohex-3-enyl)methylene]- & 36.736 & 2.9 \\
\hline 51 & Dihydro iso-jasmone & 37.154 & 0.25 \\
\hline 52 & 2-Methyl-8-nitroisoxazolizidine & 38.115 & 0.14 \\
\hline 53 & Acetic acid, 7-chloro-3-methyl-6-oxo-hept-2-enyl ester & 38.951 & 0.17 \\
\hline 54 & Cycloundecanone & 39.740 & 0.29 \\
\hline 55 & Hydrazinecarboxamide, 2-(2-thienylmethylene)- & 40.467 & 0.15 \\
\hline 56 & E-9-Methyl-8-tridecen-2-ol, acetate & 40.988 & 0.14 \\
\hline 57 & Oxalicacid, dimorpholide & 41.903 & 0.3 \\
\hline 58 & 2H-Pyran-2-one, 6-hexyltetrahydro- & 42.252 & 0.2 \\
\hline 59 & 3-Bornanone, oxime & 43.797 & 0.13 \\
\hline 60 & $\left(4 \mathrm{R}^{*}, 5 \mathrm{R}^{*}, 9 \mathrm{~S}^{*}\right)-5,9-$-Dimethylspiro[3,5]nonan-1-one & 45.045 & 0.14 \\
\hline 61 & $\begin{array}{l}\text { Methylphosphonic acid, fluoroanhydride, (2-isopropyl-5-methylcyclohexyl) } \\
\text { ester }\end{array}$ & 45.457 & 0.14 \\
\hline 62 & Hexanoicacid, 5-tridecyl ester & 45.600 & 0.36 \\
\hline 63 & Benzylalcohol, 4-methoxy-6-fluoro & 45.714 & 0.15 \\
\hline 64 & Dodecanoicacid, ethenyl ester & 45.834 & 0.21 \\
\hline 65 & Hexanoic acid, undec-2-enyl ester & 45.977 & 0.87 \\
\hline 66 & Phosphonofluoridic acid, methyl-, cycloheptyl ester & 46.441 & 0.14 \\
\hline 67 & 2-Benzimidazolinethione, hexahydro & 47.374 & 0.18 \\
\hline \multirow[t]{8}{*}{68} & Fumaricacid, di(3-hexyl) ester & 47.854 & 0.14 \\
\hline & Oxygenated monoterpenes & \multicolumn{2}{|l|}{7.78} \\
\hline & Lactones & \multicolumn{2}{|l|}{0.62} \\
\hline & Fattyacidsrelatedcompound & \multicolumn{2}{|l|}{6.91} \\
\hline & Alkaloids & \multicolumn{2}{|l|}{2.12} \\
\hline & Flavonoids & \multicolumn{2}{|l|}{1.82} \\
\hline & Aromaticcompound & \multicolumn{2}{|l|}{12.38} \\
\hline & Others & \multicolumn{2}{|l|}{68.37} \\
\hline
\end{tabular}


Abundance

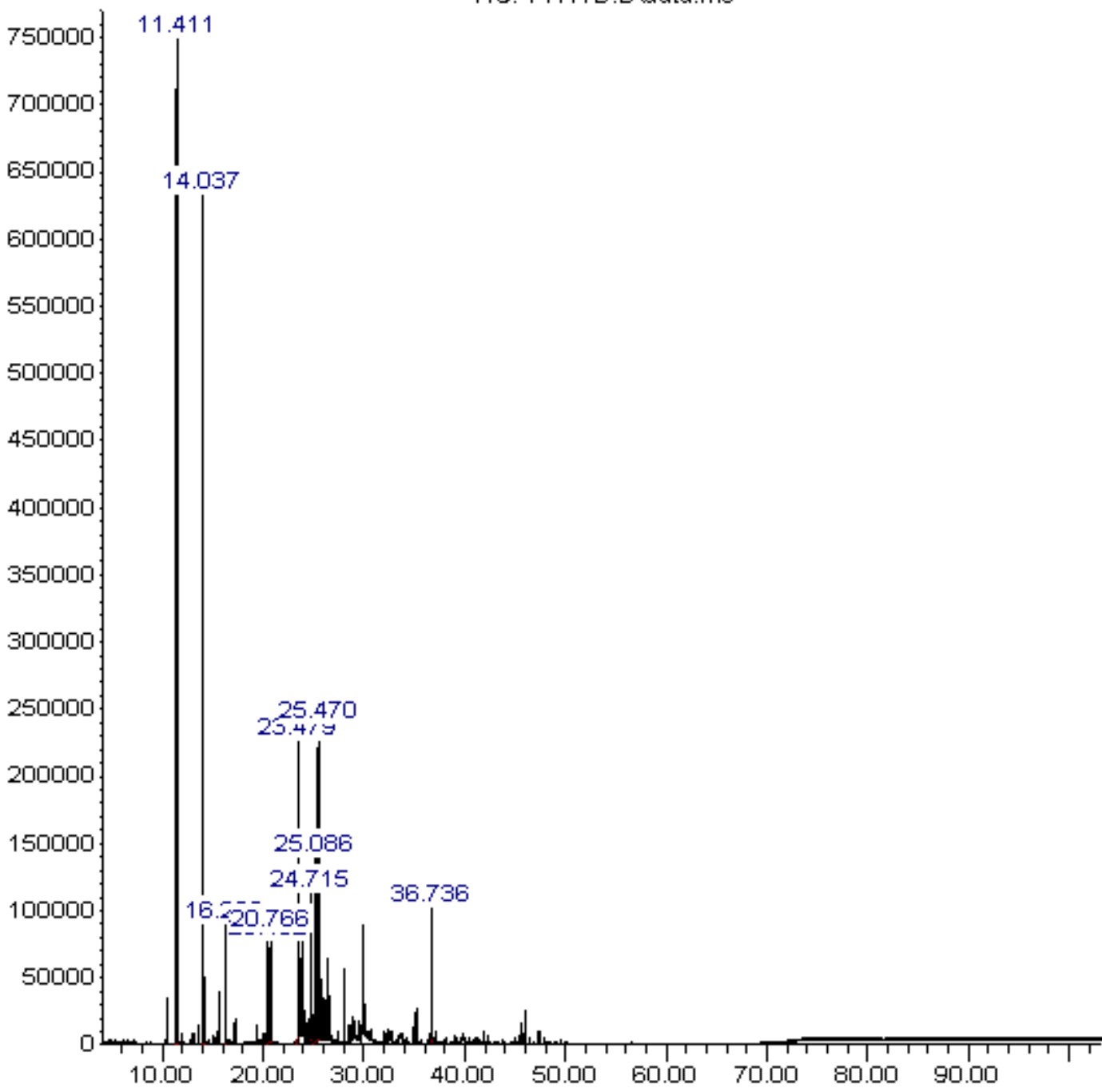

Time-->

Figure 1:- GC-MS chromatogram of essential oil of Teucrium pseudoscorodonia subsp. baeticum of north of

\section{Results and discussion:-}

Morocco.

The GC-MS analysis (table 1 and figure 1) showed that the essential oil of Teucrium pseudoscorodonia subsp. baeticum from Tangier is mainly composed of N-Formylmorpholine (25.07\%), morpholine, 4-acetyl- (17.61 \%) and 2-Oxabicyclo[2,2,2] octan-6-one, 1,3,3-trimethyl- $(6.47 \%)$. Moreover, the amount of aromatic compounds were $12.38 \%$,oxygenated monoterpenes $(7.78 \%$ ), fatty acids related compounds (6.91 \%), alkaloids (2.12\%) and flavonoids $(1.82 \%)$.

Although, the chemical composition of essential oil of Moroccan specie is completely different of Teucrium scorodonia subsp. scorodonia sample oils from Corsica and T. scorodonia subsp. Baeticum sample oils from Algeria (Djabou N. and al. 2012). The amount of oxygenated monoterpenes found in Moroccan subspecie was 7.78 $\%$ but in Corsican and Algerian Teucrium oils were respectively $2 \%$ and $4.4 \%$. Also the main components found in previous works (Djabou N., and al. 2012) were (E)- $\beta$-caryophyllene, germacrene B and $\alpha$-humulene whose not present in the Moroccan specie. 
Also, the comparison of our results with those of Maccioni S. and al. (2007) and Velasco Negueruela, A., and al. (1990) showed a total difference on the chemical composition.

These phytochemical differences could be related to genetic background, materials and method used to extract essential oil. In our investigation we extracted oil from dry leaves of the plant but in the works of Djabou N., and al. (2012) the extraction was applied on fresh aerial parts.

The obtained oil suggests an interesting application as a biopesticide because of the abundance of morpholine which derivatives are used as fungicides in cereals ergosterol biosynthesis inhibitors (Raymond, G. M., and al. 1999).

\section{Conclusion:-}

In conclusion the present work showed that Teucrium pseudoscorodonia subsp baeticum essential oil from North of morocco, have mainly components of interest like nitrogen compounds, oxygenated monoterpenes and alkaloids, which could be suitable for pharmaceutical and agricultural use.

\section{References:-}

1. Autore, G., Capasso, F., De Fusco, R., Fasulo, M. P., Lembo, M., Mascolo, N., et Menghini A. (1984). Antipyretic and antibacterial actions of Teucrium polium (L.). Pharmacological Research Communications, 16 (1), 21-29.

2. Ball, J. (1878).Spicilegium Florae MaroccanaeJ. Linn. Soc., 16, 281-772.

3. Battandier, J.A. (1917) Note sur un nouveau Teucrium de la flore marocaine.

4. Bull. (1917). Soc. Hist. Nat. Afrique N. $8: 71$.

5. Battandier, J.A. andTrabutL.C. (1890). Flore de l'Algérie et catalogue des plantes du Maroc, I. Dicotylédones. 872 p. Alger, Jourdan.

6. Cavaleiro C., Salgueiro L.R., Antunes T., Sevinate-Pinto I., and Barroso J.G. (2002). Composition of the essential oil and micromorphology of trichomes of Teucrium salviastrum., an endemic species from Portugal. FlavFrag J., 17, 287-291.

7. Cohen, E. (1956). Contribution à l'étude des Teucrium marocains de la section Polium. Tray. Inst. Sci. Chérifien. Sér. Bot. 9, 88p.

8. Cosson, E. (1873).Species novae maroccanae (serie prima). Bull. Soc. Bol.France 20. 239-261.

9. Cosson, E. (1881). Compendium Floraeatlanticaeseumethodicaplantarum omnium in Algeria... (Flore des Fiats barbaresques: Algérie, Tunisie, Maroc), I. Paris, Masson.

10. Couladis, M., Tzakou, O., Verykokidou, E., andHarvala, C. (2003). Screening of some Greek aromatic plants for antioxidant activity. Phytotherapy Research, 17, 472-476.

11. Desfontaines, A. R. (1798). Flora Allantica, 2. Paris. 923 p.

12. Djabou N., AllaliH., BattestiM.J., TabtiB., Costa J., MuselliA., VaresiL. (2012). Chemical and genetic differentiation of two Mediterranean subspecies of Teucrium scorodonia L. Phytochemistry 74, 123-132.

13. El Oualidi, J. (1987). Approche de l'étude biosystématique des Teucrium de la section Polium (Lamiaceace) du Maroc. Contribution à la "Flore Pratique du Maroc". DEA Sciences de l'Évolution et Ecologic, USTL, 91 p.

14. El Oualidi, J. (1991).Biosystématique et taxonomie des Teucrium de la section Polium (Lamiaceae) dans le bassin méditerranéen occidental. Différents aspects de la variation au Maroc, en France et en Espagne. Thèse Doct., USTL., Montpellier II, 220p.

15. El Oualidi, J. and M. IbnTattou(1993).Une nouvelle espèce du genre Teucrium section Polium, endémique du Maroc: Teucrium mitecum Ibn Tattooand El Oualidi in Acta Bol. Malacitana 18: 153-162.

16. El Oualidi, J. and T. Navarro. (1995). Position taxonomique du complexe Teucrium houtii- Teucrium grosii dans le rif marocain (Lamiacae). Anales fad. Bol. Madrid 53(2): 257-259.

17. El Oualidi, J., J. Mathezand S. Puech. (1997a)Biosystematique et variation géographique: le complexe T. huotiiEmb. and Maire (Labiatae) du Maroc. Candollea 52(1): 59-86.

18. El Oualidi, J., J. Mathezand T. Navarro (1997b).Contribution à la connaissance des Teucrium sect. Polium (Labiatae) du Maroc : le complexe de T. chlorostachyom. Fl. Medit.7: 21-26.

19. El Oualidi J., Khamar H, Fennane M., Ibn Tattou M., Chauvet S.and Taleb M.S., (2012). Checklist des endémiques et spécimens typesde la flore vasculaire de l'Afrique du Nord. Document de L’institut Scientifique, N²5, 2012, Université Mohammed V-Agdal, Rabat.

20. Emberger, L and R. Maire(1927).SpicilegiumRifanum. AVM. SOC. Sci. Nat. Maroc17: 45-46.

21. Em Berger, L and R. Maire(1941).Catalogue des plantes du Maroc, 4. Suppléments aux vols. 1, 2 and 3. Alger, Minerva.

22. Fernandez, P. B., Iglesias, P. I., and Villar del Fresno, A. M. (1997). Antiinflammatory

23. and antiulcer activity of Teucrium buxifolium. Journalof Ethnopharmacology, 55, 93-98.

24. Font Quer, P. (1928).Itermaroccanum 1927.Etiquetasnumeradas con diagnosis de novedadessobrelasetiquetas. 
25. Font Quer, P. (1932).IterMaroccanum 1930. Etiquetas numeradas con diagnosis de novedades sobre las etiquetas.

26. Franco J.A., (1984). Nova Flora de Portugal (Continente e Açores), II, Lisboa,.

27. Galati, E. M. et al. (2000). Effects of Teucrium divaricatumHeldr.ssp. divaricatum decoction on experimental ulcer in rats. Journal of Ethnopharmacology, 72, 337-342.

28. Gharaibeh, M. N., Elayan, H. H., andSalhab, A. S. (1988). Hypoglycemic effects of Teucrium polium.Journal of Ethnopharmacology, 24, 93-99.

29. Hassan M.M., Muhtadi F.J., Al-Badr A.A. (1979). J. Pharm. Sci. 68, 800.

30. Humbert, H. (1924). Végétation du Grand Atlas marocain oriental: Exploration botanique de l'Ari Ayachi. Bull. Soc. Hist. Nat. Afrique N. 15. 217.

31. Jahandiez, E. and R. Maire. (1934). Catalogue des Plantes du Maroc. Alger. Minerva.

32. Litardiere, R. deand R. Ma1re (1921). Contribution à 1'étude du Grand Atlas. Mém. Soc. Sci. Nat. Maroc IV n 120.

33. Maire, R. (1929a). Contribution à l'étude de la flore de l'Afrique du Nord, fasc. 15 (contr. 627) Mém. Soc. Sci. Nat. Maroc 21: 15.

34. Maire, R. (1931). Contribution à l'étude de la flore de l'Afrique du Nord, fasc. 17 (contr. 893); fasc. 18 (contr. 1112 , 1113). Bull. Soc. Hist. Nat. Afrique Nord 22: 62-312.

35. Maire, R. (1932). Contribution à l'étude de la flore de l'Afrique du Nord, fasc. 19 (contr. 1320, 1321, 1322, 1323). Bull. Soc. Sci. Nat. Afrique Nord 23: 208-211.

36. Maire, R. (1933). Contribution à l'étude de la flore de l'Afrique do Nord, fasc. 20 (contr. 1482). Bull. Soc. Sci. Nat. Afrique Nord 24: 297-300.

37. Maire, R. (1939). Contribution à l'étude de la flore de l'Afrique du Nord, fasc. 27 (contr. 2818,2819). Bull. Soc. Sci. Nat. Afrique Nord 30: 297-300.

38. Nagao, Y., Ito, N., Kohno, T., Kuroda, H., andFujita, E. (1982). Antitumor activity of Rabdosia and Teucriumditerpenoids against P388 lymphocytic leukemia in mice.Chemical and Pharmaceutical Bullettin, 30, 727729.

39. Navarro, T. andJ. El Oualid1 (1996). Systematic position of Teucrium tananicumMaire (Lamiaceae), rare endemic species from The Moroccan High Atlas.Acta Bot. Malacitana 21: 281-282.

40. Navarro Teresa andEl OualidiJalal(1997). Synopsis of the Genus Teucrium L. (Lamiaceae) In Morocco.Acta Botanica Malacitana 22: 187-203

41. Pau, C. (1924). Plantas del Norte de Yebala (Marruecos). Mem. R. Soc. Esp. Hist. Nat. 12: 263-401.

42. Pignatti, S. (1982). Flora d'Italia. Bologna: Edagricole.

43. Roman-Ramos, R., Flores-Saenz, J. L., Partida-Hernandez, G., Lara-Lemus, A., andAlarcon-Aguilar, F. (1991). Experimental study of the hypoglycemic effect of some antidiabetic plants.Archivos de investigacion medica, 22, 8793.

44. Rasekh, H. R., Khoshnood-Mansourkhani, M. J., andKamalinejad, M. (2001). Hypolipidemic effects of Teucrium polium in rats. Fitoterapia, 72, 937-939.

45. Raymond, G. M. andDimitrios, A. D. (1999). Evaluation of Shellac and Sucrose Ester Fruit Coating Formulations that Support Biological Control of Post-harvest Grapefruit Decay.Biocontrol Sci. Technol.9 (1), 53-65.

46. Sauvage, Ch. and J. Vindt(1955).Synopsis du genre Teucrium sect. Polium au Maroc. Bull. Soc. Sc. Nat. Phy. du Maroc 35: 283-293.

47. Sauvage, Ch. and J. Vindt(1965).Synopsis du genre Teucrium sect. Polium au Maroc (suite et fin). NaturaliaMonsp. 16: $195-200$.

48. Sauvage, Ch. and J. V1ndt (1967).Révision de l'Herbier de l'Institut Scientifique Cherifien. Notes critiques. Le Botaniste, sér. L: 415-436.

49. Sennen, E.C. (1936). Diagnoses des nouveautés parues dans les exsiccata Plantes d'Espagne et du Maroc de 1928 à 1 935. Ed. Anglada. Vic. 308p.

50. SimonettaMaccioni, Rosa Baldini, Marianna Tebano, Pier Luigi Cioni, Guido Flamini (2007). Essential oil of Teucrium scorodonia L.ssp. scorodoniafrom Italy. Food Chemistry 104, 1393-1395.

51. Tariq, M., Ageel, A. M., Al-Yahya, M. A., Mossa, J. S., and Al-Said, M. S. (1989). Anti-inflammatory activity of Teucrium polium.International Journal of Tissue Reactions, 11, 185-188.

52. Tutin, T. G., Heywood, V. H., Burges, N. A., Moore, D. M., Valentie, D. H., and Webb, D. A. (1976). Flora Europaea (Vol. 3). Cambridge: Cambridge UniversityPress.

53. Valdés B., Talavera S., Fernández-Galiano E.(1987). Flora vascular de Andaluc'́1aOcidental, Ketres Editora, Barcelona.

54. Velasco-Negueruela, A., Perez-Alonso, M., (1990). The volatiles of six Teucrium species fromthe Iberian Peninsula and the Balearic Islands.Phytochemistry 29, 1165-1169. 